\title{
Correspondence
}

Psychological Medicine, 48 (2018). doi:10.1017/S0033291717002422

First published online 29 August 2017

\section{Schizophrenia does not represent the extreme of a normally distributed trait}

In their recent opinion piece, Guloksuz and van Os point out that psychosis is continuously distributed and that subclinical levels of psychosis correlate with risk of developing schizophrenia (Guloksuz \& van Os, 2017). They accompany this with an illustration of the liabilitythreshold model, in which the trait liability follows a Gaussian distribution in the population and anybody whose liability exceeds a threshold develops the clinical syndrome. They were also authors on a recent study which applied the Community Assessment of Psychic Experiences (CAPE (Konings et al. 2006)) to a sample of patients with schizophrenia, their parents and siblings and healthy comparison subjects (van Os et al. 2017). They did not report the CAPE scores for the patients but the unaffected groups at different time points had mean CAPE-POS scores between 0.08 and 0.19 with the highest group standard deviations (S.D.) being 0.19 and mean CAPE-NEG scores between 0.39 and 0.54 with the highest group S.D. being 0.40. By contrast, a sample of German in-patients with schizophrenia had a mean CAPE-POS score of 1.86 and a mean CAPE-NEG score of 2.26 (Moritz et al. 2013). Thus, taking the S.D. of the trait as measured in unaffected subjects, patients have CAPE-NEG scores which are more than 4 S.D. higher than the population mean and CAPE-POS scores which are more than 8 S.D. higher. Likewise, as part of the Genetic Risk and Outcome of Psychosis (GROUP) study, quantitative traits related to psychosis were measured in psychosis patients, their relatives and community controls and it was found that the shape of the distributions suggested multiple component distributions (Derks et al. 2012). The relevant results are illustrated in Fig. 1. In these samples the unaffected subjects had mean scores on the positive symptom dimension of -0.54 with S.D. 0.31 and on the negative symptom dimension of -0.28 with S.D. 0.24 . In-patients and out-patients with schizophrenia had mean positive symptom scores of 0.90 and mean negative symptom scores of 0.67 , again several S.D. higher.

These results show that the distribution of psychosis may be continuous, but it is certainly not Gaussian. Furthermore, the lack of a universally clear boundary between the normal and abnormal does not mean that the concept of a discrete illness becomes invalid. Height is continuously distributed but achondroplasia is a disease and temperature is continuously distributed but many illnesses cause pyrexia. Some patients have severe schizophrenia and some have mild schizophrenia and for some the diagnosis is uncertain. One can measure psychotic traits in the general population and there may be no clear divide between patients and nonpatients. However, the fact that patients have symptom scores which are very markedly abnormal provides strong support for the argument to retain the concept of schizophrenia as an illness. (a)

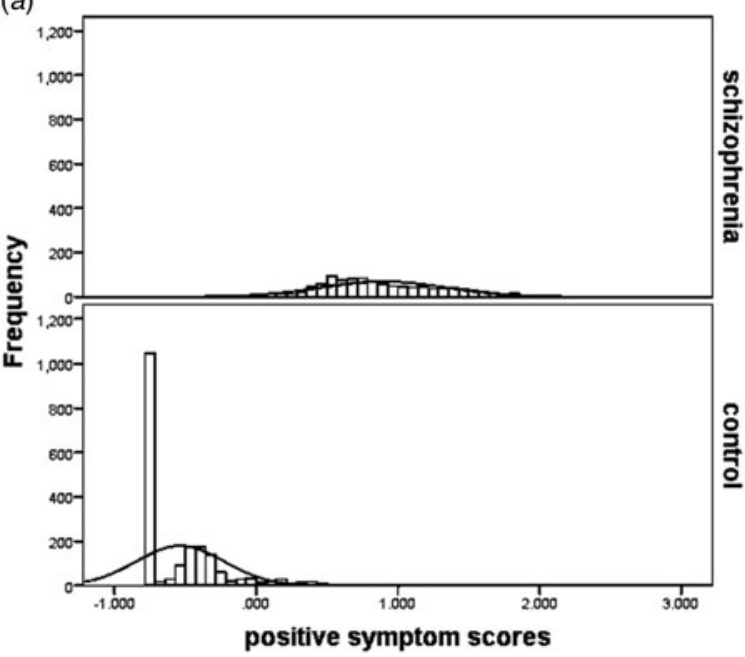

(b)

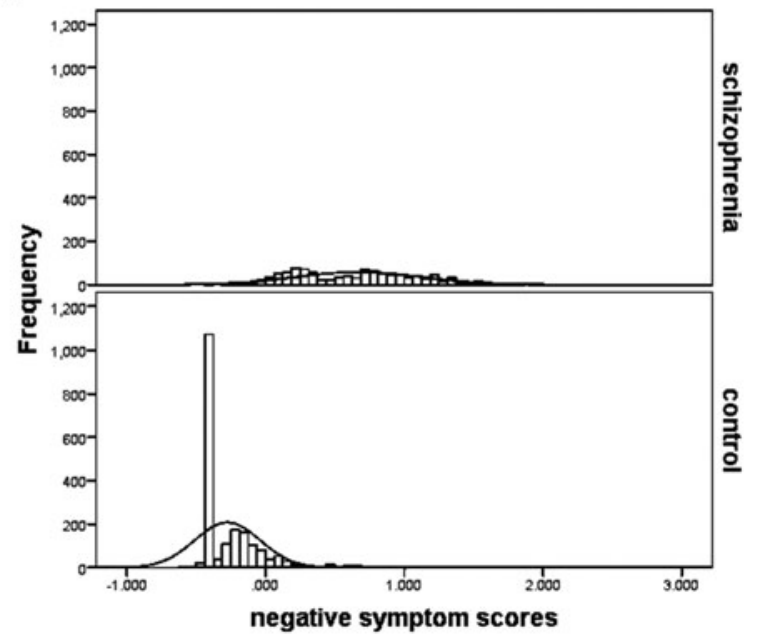

Fig. 1. Distribution of quantitative trait scores in schizophrenia patients and unaffected subjects for $(a)$ positive and $(b)$ negative symptom dimensions. 


\section{Acknowledgements}

The GROUP study was supported by the Geestkracht programme of the Dutch Health Research Council (ZON-MW, grant number 10-000-1002); the EU Seventh Framework Programme (consortium name: EU-GEI) and matching funds from participating universities and mental health care organizations (Site Amsterdam: Academic Psychiatric Centre AMC, Ingeest, Arkin, Dijk en Duin, Rivierduinen, Erasmus MC, GGZ Noord Holland Noord; Site Utrecht: University Medical Centre Utrecht, Altrecht, Symfora, Meerkanten, Riagg Amersfoort, Delta; Site Groningen: University Medical Center Groningen, Lentis, GGZ Friesland, GGZ Drenthe, Adhesie, Mediant, GGZ De Grote Rivieren and Parnassia psycho-medical centre; Site Maastricht: Maastricht University Medical Center, GGZ Eindhoven, GGZ Midden-Brabant, GGZ Oost-Brabant, GGZ NoordMidden Limburg, Mondriaan Zorggroep, Prins Clauscentrum Sittard, RIAGG Roermond, Universitair Centrum Sint-Jozef Kortenberg, CAPRI University of Antwerp, PC Ziekeren Sint-Truiden, PZ Sancta Maria Sint-Truiden, GGZ Overpelt, OPZ Rekem).

\section{Declaration of Interest}

The authors declare they have no conflict of interest.

\section{References}

Derks EM, Allardyce J, Boks MP, Vermunt JK, Hijman R, Ophoff RA, GROUP (2012). Kraepelin was right: a latent class analysis of symptom dimensions in patients and controls. Schizophrenia Bulletin 38, 495-505.

Guloksuz S, van Os J (2017). The slow death of the concept of schizophrenia and the painful birth of the psychosis spectrum. Psychological Medicine. doi: 10.1017/S0033291717001775.

Konings M, Bak M, Hanssen M, van Os J, Krabbendam L (2006). Validity and reliability of the CAPE: a self-report instrument for the measurement of psychotic experiences in the general population. Acta psychiatrica Scandinavica 114, $55-61$.

Moritz S, Van Quaquebeke N, Lincoln TM, Köther U, Andreou C, Andreou C (2013). Can we trust the internet to measure psychotic symptoms? Schizophrenia Research and Treatment 2013, 457010.

van Os J, van der Steen Y, Islam MA, Gülöksüz S, Rutten BP, Simons CJ, GROUP Investigators (2017). Evidence that polygenic risk for psychotic disorder is expressed in the domain of neurodevelopment, emotion regulation and attribution of salience. Psychological Medicine. doi: 10.1017/S0033291717000915.

D. CURTIS ${ }^{1,2, *}$ AND E. M. DERKS ${ }^{3}$

${ }^{1}$ UCL Genetics Institute, UCL, Darwin Building, Gower Street, London, UK

${ }^{2}$ Centre for Psychiatry, Barts and the London School of Medicine and Dentistry, London, UK

${ }^{3}$ Translational Neurogenomics Group, QIMR Berghofer, Brisbane, QLD, Australia

*Address for correspondence: D. Curtis, UCL Genetics Institute, UCL, Darwin Building, Gower Street, London WC1E 6BT, UK.

(Email: d.curtis@ucl.ac.uk) 